\title{
Editorial
}

\section{Non-invasive brain current stimulation in neurorehabilitation}

\author{
H. Branch Coslett ${ }^{\mathrm{a}}$ and Roy Hamilton ${ }^{\mathrm{b}}$ \\ ${ }^{a}$ William N. Kelley Professor of Neurology, University of Pennsylvania School of Medicine, PA, Philadelphia \\ ${ }^{\mathrm{b}}$ Assistant Professor of Neurology, University of Pennsylvania, PA, Philadelphia
}

Neurologic conditions such as stroke, traumatic brain injury, tumors, and degenerative diseases are often associated with dramatic impairments in brain function. Converging evidence in the fields of clinical neurorehabilitation and cognitive neuroscience has revealed that functional recovery after brain injury depends largely on compensatory plastic changes in remaining neural structures. Thus, while current treatments such as physical, occupational and speech therapy and pharmacological interventions have proven only modestly effective, there has been increasing interest in exploring potential therapies that can modulate the functioning of neural systems more directly. Noninvasive brain stimulation techniques such as transcranial magnetic stimulation (TMS), transcranial direct current stimulation (tDCS) and transorbital alternating current stimulation (ACS) are promising approaches that can potentially be employed to facilitate functional plasticity after brain injury, leading to enhanced recovery. This special issue of RNN is devoted to this topic. As will be illustrated by the contributions included here, noninvasive brain stimulation techniques have potentially important implications for rehabilitation of a variety of neurologic disorders.

The modern era of noninvasive brain stimulation was initiated by the demonstration that a magnetic field could readily and painlessly traverse the scalp and skull to generate a weak, brief and localized current in the brain (Barker et al., 1987). Subsequent studies demonstrated that, depending on the stimulation parameters, this electrical current could either inhibit or enhance neural function in the stimulated area. Over the next few years, this exciting breakthrough was exploited in investigations that contributed substantially to the development of theories regarding the localization and time course of sensory and motor processing in the intact human brain. Beginning in the $1990 \mathrm{~s}$, TMS was also used to address questions regarding the localization of "higher" cortical faculties such as language and reading.

In the late 1990's Nitsche, Paulus and colleagues demonstrated that low power (e.g., $1 \mathrm{~mA}$ ) direct current applied to the skin could alter neuronal excitability. The effects induced by transcranial direct current stimulation (tDCS) were, at least in some instances, a function of electrode polarity; that is, anodal and cathodal stimulation produced different results. Like TMS, tDCS has been used to explore motor, sensory and cognitive functions. In this issue of RNN, Nitsche and Paulus review recent progress in the use of tDCS. This comprehensive overview discusses advances in our understanding of the neural mechanisms of stimulation, reviews important refinements that have made the administration of tDCS, and surveys the variety of basic and clinical applications that have been explored in the last several years with this versatile technology. 
Recovery from motor deficits after stroke is a topic of tremendous importance in neurorehabilitation. Three contributions in this issue provide data demonstrating that non-invasive brain stimulation may be of benefit in the treatment of motor weakness resulting from stroke. Hoyer and Celnik review the ever-increasing contributions of TMS to the understanding of the mechanisms by which different motor areas contribute to recovery. They also discuss recent demonstrations that repetitive TMS may contribute to recovery of motor function in subjects with stroke. Nair, Renga, Lindenberg, Zhu, and Schlaug report a randomized, double-blind, sham controlled study of the effect of tDCS on motor function in subjects with chronic stroke; subjects received 5 consecutive days of cathodal tDCS to the contralesional motor cortex for 30 minutes while receiving Occupational Therapy. The combination of tDCS and OT provided significantly better results than sham tDCS and OT when tested one week after treatment. Furthermore, there was a correlation between improvement in motor scores and decrease in fMRI activation over the ipsilesional hemisphere. Finally, Rogers et al., review the emerging literature suggesting that non-invasive brain stimulation techniques may enhance the recovery of walking after stroke. The investigators argue that brain stimulation protocols employed in conjunction with physical therapy are likely to demonstrate compelling evidence of efficacy.

In addition to paresis, noninvasive brain stimulation is also being explored as a potential treatment for a variety of other deficits resulting from stroke. Oliveri, the first investigator to explore the potential role of TMS in the treatment of the neglect syndrome, reports data from several studies suggesting that TMS to the intact hemisphere may be helpful in the treatment of neglect; this finding is consistent with the hypothesis that over-activation of the contralesional hemisphere underlies, at least in part, the neglect syndrome. Oliveri also notes that TMS may be synergistic with other interventions such as prismatic adaptation or Occupational Therapy. Chrysikou and Hamilton review a number of studies suggesting that TMS and tDCS may facilitate language recovery after stroke. As they also note, these techniques offer important insights into the mechanisms underlying recovery of language. Song, Sandrini and Cohen provide a comprehensive review of the literature demonstrating that non-invasive brain stimulation with either TMS or tDCS can modify both low-level somatosensory function as well as multisensory integration. Although there has been little work to date exploring the potential clinical implications of this work, experience with non-invasive brain stimulation in patients with disorders of motor and language function provides reason for optimism.

Zaghi, Thiele, Pimental, Pimental and Fregni report data from non-invasive brain stimulation in a different clinical population, subjects with chronic pain. In a meta-analysis of 18 studies in which non-invasive brain stimulation was employed as a treatment, they found that chronic pain was significantly ameliorated by non-invasive brain stimulation. Furthermore, as they note, non-invasive brain stimulation can provide important insights into the pathophysiology of chronic pain states.

A growing body of work also suggests that tDCS and related techniques can be employed to enhance neural activity in the visual system, with potentially beneficial effects for patients with visual deficits mediated by lesions of the central nervous system. Antal and Nitsche review data from tDCS as well as trancranial Alternating Current Stimulation (tACS) investigations of visual processing in normal subjects and clinical populations. Their work elegantly demonstrates that both techniques can selectively influence different components of visual processing. Olma and colleagues provide additional experimental evidence demonstrating the ability of noninvasive brain stimulation to enhance visual processing, employing signal detection theory (SDT) to demonstrate that anodal stimulation of the visual cortex in normal subjects results in improved detection sensitivity for visual targets. Finally, Sabel and colleagues report exciting results of a prospective, double-blind, randomized, placebo-controlled clinical trial in which patients with chronic optic neuropathy who were treated for 40 minutes daily for 10 days with transobital ACS experienced a $69 \%$ reduction in visual field detection deficit as well as significant improvement in visual processing speed, detection of targets in static perimetry, and visual acuity. These changes were stable two months after treatment.

The contributions to this special issue make a compelling case for the value of non-invasive brain stimulation for rehabilitation. Although there is an urgent need to address questions such as the optimal stimulus location and parameters as well as subject selection, the ability of techniques such as TMS, tDCS or tACS to modify neuronal function and enhance brain plasticity offers substantial promise for the development of new therapies for many devastating brain disorders. 


\section{References}

Antal, A., Paulus, W. \& Nitsche, M.A. (2011). Electrical stimulation and visual network plasticity. Restor Neurol Neuros, 29, 367376.

Barker, A.T., Freeston, I.L., Jalinous, R. \& Jarratt, J.A. (1987). Magnetic stimulation of the human brain and peripheral nervous system: An introduction and the results of an initial clinical evaluation. Neurosurgery, 20, 100-109.

Chrysikou, E.G. \& Hamilton, R.H. (2011). Noninvasive brain stimulation in the treatment of aphasia: Exploring interhemisperic relationships and their implications for neurorehabilitation. Restor Neurol Neuros, 29, 377-396.

Hoyer, E.H. \& Celnik, P.A. (2011). Understanding and enhancing motor recovery after stroke using transcranial magnetic stimulation. Restor Neurol Neuros, 29, 397-411.

Nair, D.G., Renga, V., Lindenberg, R., Zhu, L. \& Schlaug, G. (2011). Optimizing recovery potential through simultaneous occupational therapy and non-invasive brain-stimulation using tDCS. Restor Neurol Neuros, 29, 413-422.

Nitsche, M.A. \& Paulus, W. (2011). Transcranial direct current stimulation - update 2011. Restor Neurol Neuros, 29, 467-496.
Oliveri, M. (2011). Brain stimulation procedures for treatment of contralesional spatial neglect. Restor Neurol Neuros, 29, 423427

Olma, M.C., Kraft, A., Roehmel, J., Irlbacher, K. \& Brandt, S.A. (2011). Exitability changes in the visual cortex quantified with signal detection analysis. Restor Neurol Neuros, 29, 457-465.

Rogers, L.M., Madhavan S., Rothe H. \& Stinear J.W. (2011). Transforming neurorehabilitation of walking following stroke: The promise of non-invasive brain stimulation - a review Restor. Neurol Neuros, 29, 511-520.

Sabel, B.A., Fedorov, A.B., Naue, N., Borrmann, A., Herrmann, C. \& Gall, C. (2011). Non-invasive alternating current stimulation improves vision in optic neuropathy. Restor Neurol Neuros, 29, 497-509.

Song, S., Sandrini, M. \& Cohen, L.G. (2011). Modifying somatosensory processing with non-invasive brain stimulation. Restor Neurol Neuros, 29, 429-439.

Zaghi, S., Thiele, B., Pimentel, D., Pimentel, T. \& Fregni, F. (2011). Assessment and treatment of pain with non-invasive cortical stimulation. Restor Neurol Neuros, 29, 441-453. 